\title{
Filigrane
}

Écoutes psychothérapiques

\section{Extraviada : égarée. À propos de Je l'ai tué, dit-elle, c'est mon père, de Raquel Capurro et Diego Nin}

\section{Prado de Oliveira}

Volume 16, numéro 1, printemps 2007

Les hauts lieux et non-lieux du rêve I

URI : https://id.erudit.org/iderudit/016182ar

DOI : https://doi.org/10.7202/016182ar

Aller au sommaire du numéro

Éditeur(s)

Revue Santé mentale au Québec

ISSN

1192-1412 (imprimé)

1911-4656 (numérique)

Découvrir la revue

Citer ce compte rendu

de Oliveira, P. (2007). Compte rendu de [Extraviada : égarée. À propos de Je l'ai tué, dit-elle, c'est mon père, de Raquel Capurro et Diego Nin]. Filigrane, 16(1),

123-131. https://doi.org/10.7202/016182ar d'utilisation que vous pouvez consulter en ligne. 


\title{
Extraviada: égarée. À propos de Je l'ai tué, dit-elle, c'est mon père, de Raquel Capurro et Diego Nin ${ }^{1}$
}

\author{
prado de oliveira
}

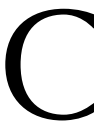

'est une jolie maison, remplie de plantes vertes, dans une rue calme, encore un peu éloignée du centre, à Montevideo. Le quartier n'est pas encore urbanisé, voire il est loin de l'être. Quelques voisins, beaucoup d'oiseaux au printemps et en été, des saisons très européennes. L'Uruguay a été longtemps considéré comme une sorte de Suisse latino-américaine.

Je reprends la quatrième de couverture. C'est l'été dans l'hémisphère sud, décembre 1935. Un coup de pistolet secoue la ville: Iris, jeune et brillante étudiante, tue son père, Lumen Cabezudo. Raimunda Spósito, sa mère, soutient sa fille. La version maternelle des événements est largement accueillie. La fille se fait l'écho de sa mère. Un père tyran, menaçant, violent, sadique, largement incestuel, sinon incestueux, bizarre de tout point de vue. Une mère victime. Des petits enfants accablés. La fille aînée délivre la famille de ce monstre. Elle dit simplement : «Je l'ai tué. C'était mon père. » Une chose justifie l'autre.

Lumen Cabezudo. Lumen Cabezón. Lumière têtue. Feu têtu. Un nom à paranoïaque. La remarque a été faite: très souvent dans les psychoses, un nom, signifiant particulier, qui ne devrait avoir d'autre signifié qu'un sujet, échappe à cette règle, colle au monde des signifiés.

Les choses n'y sont pas encore. Pour l'instant, c'est un non-lieu. Iris a tué sous la menace de la folie meurtrière de son père. Cela couvait depuis longtemps. Lorsqu'il y avait des crimes, où un homme tuait une femme, Lumen les commentait en famille, avec des remarques sympathiques au sujet des meurtriers. Au moment du crime, il s'était emporté. Il avait juré qu'il allait tuer Raimunda, tuer toute sa famille. Il était parti chercher une arme, il était revenu, il était agité.

Libérée après une année de prison, Iris revient dans sa famille, reprend ses études, les termine, devient institutrice. Sa photo fait penser à une fille, ce caractère ferme, entier, décidé.

L'enfer familial néanmoins ne prend pas fin avec la disparition du père. Au contraire. Raimunda multiplie les reproches à l'égard du défunt. Octave Mannoni a signalé ces querelles qui se poursuivent au-delà de la mort. Freud a oublié les paranoïaques quérulents, ceux qui éprouvent un impératif passionné de discuter, 
de débattre, d'accuser, de se défendre. Les psychanalystes aussi les ont oubliés. Et pour cause.

Iris découvre: mais c'est sa mère qui est folle ! En décembre 1956, 21 ans après le meurtre de son père, elle demande une expertise de sa mère. Ce qui l'amène, elle, la libératrice, à l'hôpital psychiatrique. Les réactions des psychiatres lui étaient imprévisibles. Elle essaie de s'en expliquer par écrit. Elle est libérée de l'hôpital, mais à deux conditions : quitter la maison familiale et accepter sa mise à la retraite. Elle devient vagabonde, presque sans aucun appui.

La querelle ne se réduit plus au giron familial. Elle s'étend. Iris critique l'enseignement national, les dangers liés à l'intrusion des catholiques dans l'école laïque, elle se méfie de ceux, rarissimes, qui l'accueillent encore.

Elle aurait été en Uruguay ce qu'ont été en France les sœurs Papin. Mais pourquoi comparer? Car ces sœurs n'avaient pas le talent littéraire d'Iris, ni ontelles eu des carrières d'enseignantes, leur affaire a concerné plutôt le meurtre de la mère, en premier lieu, leur patron ne se promenait pas dénudé dans la maison. La comparaison ici cacherait l'aveu de l'ignorance, mais il est inutile d'insister encore sur l'aveuglement hexagonal sur ce qui se passe dans le reste du monde. Dommage que les psychanalystes français n'aient pas connu l'histoire de la famille Cabezudo dès ce crime. Autant de raisons d'être reconnaissants envers ce que les traducteurs apportent et envers Christine Angot.

Il y a sans doute de bonne raisons pour un changement de titre. En espagnol - et il est inutile de préciser uruguayen ou argentin -, le titre de ce livre est d'une remarquable économie. Thrift, Horatio, thrift! - rappelait Freud. Extraviada: égarée. C'est un des sens possibles de la folie ou de la paranoïa ou de la paraphrénie. Les sens des mots ont été oubliés.

Je quitte la quatrième de couverture de ce livre si riche. Je viens au sens des mots. Les mots paranoïa ou paraphrénie ont été appropriés par la psychiatrie comme des nouveaux riches essayent de s'attribuer des ancêtres illustres. Les psychanalystes les ont repris pour se donner des airs de psychiatres. La psychiatrie ou la nosographie, la clinique supposée différentielle, maladies infantiles de la psychanalyse. La nosographie est aussi utile à la psychanalyse que l'astrologie à l'astronomie.

Paraphrénie est un terme employé par Hérodote. Il ne veut rien dire d'autre que «à coté de ses esprits». Paranoïa est un mot utilisé par Eschyle et par Euripide. Edipe et Jocaste sont paranoïaques du fait de leur union. Oreste est paranoïaque du fait du meurtre de sa mère, Clytemnestre. Ils sont «à côté de leurs connaissances ». D'ailleurs, ils seront pardonnés. Hippocrate a volé ce mot aux poètes. Il en a fait une affaire de rougeurs de gorges qui s'étendent vers l'intérieur du corps. Il n'est pas tellement question ici d'une quelconque dualité entre l'esprit et le corps, mais plutôt de la création par la médecine naissante d'une pseudo réalité pour cacher son ignorance foncière d'une parole qui circulait et s'envolait dans le domaine des commémorations liées à la tragédie.

Platon et Aristote aussi utilisent le vocable paranoïa. Sont paranoïaques parents et enfants qui ont des mauvaises relations, surtout quand les parents sont âgés et 
que les enfants veulent s'approprier de leur héritage. Sont paranoïaques ceux qui se révoltent contre les traditions. Ainsi, par un curieux tournant des choses, Platon considère paranoïaques ceux qui ne croient pas à la vie après la mort et ceux qui ne croient pas à l'intervention des morts dans les affaires courantes des vivants. Ses raisons sont simples: la tradition, le fait que ce sont des croyances bien établies, que de tout temps, selon lui, les hommes y ont cru.

Paraphrénie est un mot léger par rapport à paranoïa, et les deux, par rapport à la manie ou à la fureur, mots qui désignent la folie chez les grecs anciens. Paraphrénie veut dire simplement égaré, mais pas très loin. L'esprit y est encore.

Paranoïa était un mot populaire en Allemagne. Vers la deuxième moitié du $\mathrm{XIX}^{\mathrm{e}}$ siècle, les aliénistes ont voulu lui donner une dimension scientifique. Ziehen l'utilise pour dire la folie. Kræpelin a voulu créer des catégories bien définies. Il utilise paraphrénie pour dire ce qui deviendra avec Bleuler, d'abord, Melanie Klein ensuite, la schizophrénie paranoïaque ou paranoïde. C'est le pain quotidien de ceux qui travaillent auprès des fous. Rien de plus commun que la paraphrénie, ou la schizophrénie paranoïaque, dans le royaume des hôpitaux psychiatriques ou autres centres de soin.

Iris Cabezudo aurait commencé paranoïaque, autant qu'Edipe ou Oreste. Elle a évolué vers la paraphrénie, au sens de Kræpelin. Iris n'est pas Schreber. Le paranoïaque peut tuer, il le fait souvent. Ce geste, chez le paraphrénique, est bien plus rare. En tout cas, il y a en un qui se heurterait à la loi de la cité; il y en a l'autre qui la défendrait. Ce ne sont pas les mêmes. Ou bien, si, mais à différents moments de leur existence. Rarement au même instant, même si cela peut exister. C'est là, alors, une forme de perversion, la perversité.

Mais il y a un point commun entre Iris, la têtue, et Schreber. Tous les deux sont issus de nids de paranoïa. Inutile de reprendre ici la remarquable histoire de la famille Schreber, depuis un ancêtre curé et auteur de nouvelles grivoises, jusqu'à un père fou de gymnastique et une mère dont l'esprit était rempli bien plus qu'il ne le fallait de culture, selon les mots du Président lui-même, qui ne manquait pas d'esprit critique ${ }^{2}$.

Et Iris, cette histoire à rebondissements qui nous garde en haleine, égarés. Il faut la lire, pour comprendre la fabrique de la folie. Ce livre présente les articles de presse, les textes d'Iris, les déclarations de sa mère et de son frère, les témoignages, les dépositions, les jugements, les expertises médicales, les archives, la lutte de ses collègues de classe pour la soutenir, les pièces culturelles, poèmes ou romans, qui marquaient l'époque, les dossiers de l'Éducation nationale, où Iris a travaillé, les souvenirs de ceux qui l'ont connue à la fin de sa vie, tout cela remarquablement réuni et commenté par Raquel Capurro et Diego Nin.

Voilà Iris qui marche dans les rues de Montevideo, d'un pas rapide et ferme, été comme hiver, très sobrement habillée, vers l'école où elle fait ses études, puis, passionnée vers son travail, des années plus tard, chancelante, habillée en loques, mais toujours propre, traînant avec elle ces sacs indescriptibles que les fous traînent avec eux souvent, témoins d'une sorte de carapace. Et notre désespoir: 
voilà l'humanité. Un jour, elle a été une brillante étudiante. Plus qu'un exposé de cas, magnifique, c'est un roman, qu'organisent Capurro et Nin, au sens où le voulait Freud de ses «contes de Noël» ou «fantaisie d'un printemps» à travers lesquels la métapsychologie devient vivante.

\section{Nid de paranoïaques}

Lumen, le père d'Iris, est un illuminé. En Uruguay, en 1930, il croit déjà à des spiritualités bouddhiques et au naturisme, qu'il pratique à la maison et qu'il impose à ses enfants. Il abuse d'une de ses filles et pourquoi n'aurait-il pas abusé d'Iris au-delà de l'imposition du naturisme ? Il lit des revues pornographiques, qu'il laisse traîner ici et là, à la portée des enfants. C'est un pervers.

La perversion dans la paranoïa, au-delà de la perversité, porte des traits particuliers. Elle correspond à un souci d'amener l'autre à conduire sa vie selon les critères du paranoïaque et non pas selon ses critères propres. La perversion paranoïaque correspond à une tentative de maîtrise de la vie d'autrui, ou de situations qui le concernent, sans aucun souci à son égard. Meurtre d'âme ${ }^{3}$.

Schreber s'impose en tant que femme à sa propre femme. Sa paranoïa a évolué vers la paraphrénie. Lumen s'impose nu dans la vie quotidienne de la maisonnée. Il s'allonge nu dans un hamac pendu à l'arrière-fond de la maison pour lire son journal.

Au travail, ses relations sont marquées du sceau de la perversité. Il essaie de nuire à ses collègues et de se mettre en valeur, il s'en vante sans aucun scrupule à la maison, auprès de sa femme et de ses enfants. Aujourd'hui, cette pathologie est devenue courante : nuire à son prochain avec amitié et bonnes intentions ${ }^{4}$. Surtout ne pas s'en excuser. Les excuses témoigneraient d'une certaine reconnaissance. À exclure.

Dans son couple, Lumen impose des dessins et des revues pornographiques à sa femme. Il exige d'elle des choses qu'elle considère inadmissibles. Il les lui impose de manière sadique. Il la bat, violemment, sauvagement, il lui fend le cuir chevelu en lui cassant sur la tête des cendriers ou ce qu'il trouve à sa portée. Elle doit feindre avoir tombé dans l'escalier. Il est même incroyable qu'elle ait survécu. $\mathrm{Ou}$, alors, les masochistes paranoïdes ont des ressources physiques remarquables.

Car Raimunda est aussi très particulière. Elle était venue faire des études en Europe, ce qui la mettait en valeur parmi les femmes de sa génération. Un brillant avenir lui était réservé, tout comme à sa fille, plus tard. La veille de son départ, ou le jour même, Lumen fait sa connaissance lors d'une conférence au sujet de thèmes ésotériques. Il la veut comme épouse. Elle se refuse. Il lui promet d'attendre des années, s'il le faut et, en effet, il attendra. Elle le décourage. À son retour de voyage, elle change d'avis.

Mais quelle folie a donc saisi cette femme? Que n'a-t-elle pas vu l'éclat du regard de Lumen, son agitation, sa nervosité ? Pour lui, elle abandonne tout ce à quoi elle est promise. Mais elle reste sa supérieure intellectuelle et culturelle. Et elle lui fait savoir. Elle le provoque même, armée de cet outil. Et il le reconnaît. Il l'admire à 
l'extrême. S'ils ne partagent pas grand-chose, ils partagent au moins leurs croyances bouddhiques. Et la violence. Il la bat, elle l'accuse, elle crie, elle le dénigre.

Notre culture a choisi Sacher-Masoch comme porte-étendard du masochisme. Peut-être. Mais la lecture attentive de La Venus à la Fourrure montre que l'histoire de ses souffrances aux mains de Wanda est en vérité un apologue qui sert à justifier la domination complète des femmes par les hommes. Tel est le masochisme paranoïaque: la souffrance physique de l'un lui sert à justifier la torture morale qu'il inflige à l'autre et, quand le premier passe au terrain de l'imposition d'une souffrance morale, le second imposera une souffrance physique.

Dans la dynamique propre au masochisme paranoïaque, la femme qui souffre est idolâtrée et l'homme qui bat est amoindri. Le véritable masochisme est plutôt celui de Wälser et de L'Institut Benjamenta. Wälser, tête à claque, n'est en rien valorisé et s'achemine doucement, mais sûrement, vers sa folie.

Le pur masochisme, s'il existe, correspond à une souffrance silencieuse. Le masochisme hystérique est constitué d'une souffrance scandaleuse. Le masochisme paranoïde, lui, se définit par une souffrance induite accompagné d'accusations. La douleur normale exige une fuite ou une protection, mais exclut sa perpétuation.

Raimunda, en vérité, ne fait rien pour se protéger et protéger ses enfants. Quant à la fuite, si elle y songe un moment, elle se fie à une sorte de tribunal familial séquentiel. Elle exhibe sa souffrance aux rares familiers qui viennent les visiter. D'ailleurs, l'isolement de cette famille Cabezudo est remarquable. Pas un homme pour défendre Raimunda, pas une femme pour la conseiller. Pas une femme pour discuter avec Lumen, pas un homme pour le corriger! L'isolement est, lui-même, signe pathologique.

Le masochisme de Lumen le mène à chercher la mort. C'est lui qui amène à la maison l'arme qui servira à le tuer. Et il l'oublie. Il la laisse à sa femme, qui la cache. Et qui fait de leur fille, Iris, la dépositaire du secret de la cache de cette arme.

\section{Iris}

Pas un sourire. Pas le temps de rire. Que de la passion! Que son ambition pure et réformatrice, toute tendue vers le salut, des siens, d'abord, des étudiants ensuite.

«Depuis mes plus lointains souvenirs, depuis que j'étais enfant, nous vivions à la maison dans la sensation d'une profonde crainte que nous inspirait notre père. Dernièrement, ce sentiment s'est transformé en terreur qu'il continuait à nous inspirer. Je n'ai jamais connu la chaleur ni la douceur, ni le refuge moral que procure un foyer. Et je dis que cela est en relation et est la conséquence de la conduite et de la façon d'être de mon père, alors qu'au contraire ma mère est une sainte que j'adore. » Iris Cabezudo, le 12 décembre 1935. Mais d'où tenait-elle que les foyers ne s'éteignent jamais?

«Je n'avais pas de haine envers lui. Absolument pas. Quand je voyais les regards, de haine parfois, de convoitise, d'autres regards encore, avec lesquels il regardait maman, cela m'agaçait beaucoup, me fâchait et avec raison (n'importe quelle personne droite qui aurait assisté aux scènes qui se déroulaient à la maison 
se serait indignée); mais quand je le voyais fatigué, ou soucieux ou quand il était distrait sans penser à des mauvaises choses, il me faisait pitié parce qu'on voyait que c'était une personne sans valeur propre: il était mauvais oui, très mauvais; mais il était mauvais pour cela même, parce qu'il n'avait pas de valeur positive, il n'avait pas de raison de vivre, et il s'était proposé de s'élever (grâce à ce que les autres possédaient), spécialement avec ce qui était le fruit du travail, de l'intelligence et de l'abnégation de maman, de maman qui était la personne qu'il tenait en son pouvoir, enchaînée.» Iris, le 16-17 décembre 1935.

La voilà suspendue au regard de son père, s'abreuvant de sa haine, de sa fatigue, de ses soucis, de son manque de valeur, devinant ses pensées. Et pourquoi sa mère était-elle «enchaînée»? Pour trois raisons: elle, qui avait étudié en Europe et appris tant de bonnes et belles choses, ne pouvait se résigner à voir sa maison s'effondrer; ensuite, afin que ses enfants ne connaissent pas la souillure de cet effondrement; enfin, parce que si Lumen pouvait comprendre qu'elle avait triomphé, il la tuerait. C'est la trilogie paranoïaque: la mégalomanie liée à la souillure, le meurtre. Iris ne comprend pas que sa mère introduit le meurtre tout autant que son père. Quant à l'effondrement, il s'agît plutôt de ne pas le reconnaître, de ne pas se rendre à l'évidence, de prétendre rester sourde au-delà des cris et des injures. Cela aurait complété le tableau: son quatrième bord.

Mais il y a les rebondissements: "Ce fut alors que (peut-être en 1944), avec l'appui total de mon frère cadet Lumen (qui, si c'était cette année-là, avait 16 ans), je lui demandai à grands cris (car c'était la seule façon de parler que maman entendait) qu'elle ne me parle plus de papa, qu'elle n'avait pas le droit de le faire, que ça me rendait nerveuse, qu'elle gâchait notre vie...

«À partir de ce moment maman commença à me regarder avec méfiance, et quelques mois passèrent... le thème revint. Avec la même impétuosité, en l'évoquant de manière identique quelle qu'en soit la raison, avec la même absence totale de respect pour nos vies (celle de ses enfants) nous qui luttions pour nous libérer de la crainte, et être normaux, et être heureux.

«Et c'est ainsi que j'en arrivais à la conclusion (pour moi déconcertante) que la haine de ma mère pour mon père est inextinguible: elle ne s'est éteinte ni avec sa mort, ni avec son discrédit (devenu presque total). Elle le hait aujourd'hui avec la même fureur qu'en 1935. Elle n'oublie à aucun moment de vilipender le père... » Iris, 1957. Elle a l'intuition de quelque chose qu'elle ne peut pas comprendre pleinement, qu'elle ne peut pas abréagir, comme elle écrit. C'est qu'avant d'être haine envers son père, et même envers ses enfants, c'est un pur jaillissement de haine, sans objet, vers ce que l'on pourrait considérer comme la pulsion de mort.

Certes, cette haine probablement existait aussi chez Lumen. Ce couple s'était formé dans la haine, uni par la haine, enfanté dans la haine. «La haine précède l'amour», écrit Freud. La voilà dévoilée.

Pourtant, Iris le lit et elle ne veut pas le comprendre. Elle l'accuse de simplisme. Elle tient à la force de l'amour. Elle ne peut pas comprendre que l'amour toutpuissant se transforme en haine, ou, plutôt, redevient de la haine. 
Lumen, le petit frère, veut aller au bal. Sa mère veut le lui interdire. «Regarde, tu as besoin de toujours persécuter quelqu'un: d'abord papa que tu persécutais et harcelais sans nécessité, exagérant les choses, et ensuite Ariel "qui ne te laissait pas vivre" et je t'ai crue, mais maintenant c'est terminé, tu ne vas pas persécuter Lumen. Lumen n'est pas ton mari qui te surveille et t'enferme, ce n'est pas Ariel qui te critique, Lumen est bon, il est joyeux, il est sain, il est beau, c'est un très bon étudiant, tout le monde l'apprécie, il n'a pas de vices ni de mauvaises tendances, tout le monde l'aime... [...] Tu vas laisser Lumen en paix. Si tu ne voulais pas avoir d'enfants qui ressemblent à papa, tu n'aurais pas dû te marier avec lui. [...] Qu'il aille au bal, qu'il ait une fiancée et qu'il se marie!» - se souvient Iris.

«Maman ne me répondit rien, mais depuis ce jour elle tourna contre moi toute sa rancœur. Ce jour-là je forgeais ma destruction... à coup sûr ?» souligne Iris. Elle venait de voir un film dont le titre était La haine qui fut l'amour. Ce film l'avait beaucoup troublée. Elle ne parvenait pas à comprendre ce mélange. Mais, dans son discours à sa mère, dont elle découvre la paranoïa, des enfants qui ressemblent à papa ? Comment le comprendre: la description qu'elle donne de son petit frère Lumen est celle d'une ressemblance à son père ? D'un père d'autrefois, d'un père aimé, joyeux, bon, sain? D'un père d'avant d'être pris dans le discours de la mère? D'un père d'avant d'être devenu violent, méchant, mauvais ?

Les choses se confondent dans l'esprit d'Iris. Les odeurs apparaissent. Impossible de savoir si ce sont des hallucinations ou la description de faits perçus. D'ailleurs, quelle importance. Le meurtre du canari, oiseau de discorde final entre ces deux femmes, précipite Iris dans une autre dimension, celle du meurtre et de la folie.

Iris marchait dans la rue dans des jupes plissées, avec un grand sac, dans lequel elle portait ses papiers — tout ce qu'elle écrivait —, et une tresse dans le dos. Cette tresse, elle la gardait depuis son plus jeune âge. Peut-être le sac aussi, autrement, comme un cartable, par exemple.

La chambre où Iris finit ses jours: tout y faisait peur. Partout, des piles de journaux, des marmites... de tout. Rideau.

\section{Remarques}

Toute traduction a ses problèmes: peut-être diktat rend mieux la pensée du traducteur, mais cela fait perdre la banalité, le caractère quotidien pour une écolière de dictée, dictado. Un certain nombre de choix douteux existent dans cette traduction.

Une traduction constitue déjà une interprétation. L'essentiel est le résultat du travail accompli, son effort et sa délicatesse, pleinement présents ici sous la plume de Françoise Ben Kemoun.

Il y a aussi des questions de fond, psychanalytiques, qui méritent recherche, débat, réflexion, et que, à mon avis, Raquel Capurro et Diego Nin abordent avec une certaine légèreté. 
Au sujet du psychiatre d'Iris, ces auteurs écrivent: «L'obstacle qu'il rencontre n'est pas éloigné de celui que suggère Freud dans le traitement de la psychose, en déclarant que la psychanalyse est impossible pour un psychotique parce que celuici n'entre pas dans le transfert. Il faudra attendre l'avancé proposée par Lacan pour reconsidérer la théorie freudienne du transfert et du narcissisme, et situer l'expérience particulière du transfert psychotique » (Capurro et Nin, 2005, 337).

Entrons-nous en religion? Oublions-nous l'histoire de la psychanalyse? D'abord, «l'impossibilité » attribuée à Freud n'est nullement attestée dans le texte freudien. La plupart des cas exposés dans les Études sur l'hystérie connaissent une évolution rapidement psychotique, ce qui n'empêche pas Freud de les traiter. Les considérations sur le transfert ne sont pas absentes des études freudiennes sur le président Schreber. Freud ne nie pas, mais au contraire salue, les avancées de Karl Abraham sur les psychoses maniaco-dépressives. Bien avant Lacan, Melanie Klein propose une théorie précise du transfert psychotique ${ }^{5}$. Telle ou telle remarque de Freud sur ses difficultés avec les psychotiques mériterait d'être située avec précision.

Ou bien : «En 1955, Lacan donne au délire le statut de phénomène élémentaire en soulignant qu'“il s'agit toujours de la même structure, comme on peut le voir dans une plante soit que l'on examine celle-ci en totalité soit que l'on considère seulement la feuille. Le délire ne se déduit pas (des phénomènes élémentaires) mais reproduit leur force constitutive ; il est lui aussi phénomène élémentaire.» Et les auteurs concluent, plus prudents: «Selon ce point de vue, l'absence de délire en relation avec l'acte d'Iris ne disqualifie pas cet acte comme acte paranoïaque..." » (Capurro et Nin, 2005, 355).

Encore faut-il questionner Lacan, le faire travailler, plutôt que de le prendre comme parole d'évangile. Est-ce que le fait que le délire reproduirait, car il est difficile de savoir s'il le fait vraiment, la force constitutive du phénomène élémentaire suffit à le qualifier en tant que tel ? Souvent l'attention aux structures fait oublier l'attention à l'histoire, c'est-à-dire, à ce que dans la théorie comme dans le cas clinique est de l'ordre d'une dynamique.

Je ne pense pas que Freud reprenne à son compte les thèses d'un Bleuler au sujet des phénomènes élémentaires dans les psychoses. En revanche, Freud avance une thèse qui implique à coup sûr une dynamique, une économie et une topique, soit ce qu'il nomme comme condition essentielle de toute démarche psychanalytique: une métapsychologie. Cette thèse est celle du refoulement et du retour du refoulé. Il discute avec Ferenczi au sujet de leur articulation. Freud se pose la question de savoir si refoulement et retour du refoulé se déroulent pareillement, empruntent les mêmes voies, avec la même violence dira Lacan. Et Freud répond que non ${ }^{6}$.

Nous ne pourrons jamais savoir si Lacan était au courant de cet échange entre les deux pionniers de la psychanalyse. Balint le connaissait, puisqu'il gardait les lettres de Freud à Ferenczi. Mais, à supposer que Lacan ne l'ait pas connu, sa grande perspicacité lui aurait permis d'entrevoir l'enjeu des thèses publiées par 
Freud dans son étude sur Schreber, et c'est tout à son honneur. Malheureusement, pris dans des enjeux psychiatriques de l'instant, saisi d'une curieuse intuition botanique, il marque de l'empreinte de Bleuler ses thèses sur l'approche psychanalytique des psychoses.

Qui peut savoir si le délire d'Iris n'était déjà pas là, latent, au moment de son geste parricide, devenant manifeste au cours des années? En tout cas, la sousculture de la famille Cabezudo semble bel et bien atypique. Raimunda en donne une preuve éclatante: Lumen vivait à l'ombre de devises, étiquettes dans un rayonnage: des «il faut» et «il ne faut pas»(Capurro et Nin, 2005, 121). Leur fille n'y a pas échappé.

Remercions Capurro et Nin de relancer les réflexions et les recherches en apportant cette dramatique et exemplaire histoire de notre condition humaine.

prado de oliveira

107 , rue mouffetard

75005 paris

pradodeoliveira@free.fr

\section{Notes}

1. Je l'ai tué, dit-elle, c'est mon père, Raquel Capurro, Diego Nin, traduit de l'espagnol (Uruguay) par Françoise Ben Kemoun, 2005, Paris, Epel, Monographie Clinique, 380 p., Envoi de Christine Angot.

2. Voir aussi le beau Schreber Président, sous la direction de Petitjean, Smith \& Thiellement, Fage Éditions, 2006.

3. Voir Prado de Oliveira, Schreber et la paranö̈a: le meurtre d'âme, L'Harmattan, 1996.

4. A. Honneth, La société du mépris : vers une nouvelle théorie critique, La Découverte, 2006.

5. Voir le très important livre de M.-C. Thomas, Étude des concepts kleiniens dans l'œuvre de Jacques Lacan: conséquences pour la psychanalyse d'enfant, ANRT Diffusion, 2001. Il ne s'agit pas seulement des conséquences pour la psychanalyse d'enfants, mais plutôt de conséquences pour la psychanalyse tout court, en général, dans la mesure où il s'agît d'un premier pas pour historiciser la pensée de Lacan et la sortir du type de religiosité où elle est le plus souvent inscrite.

6. Lettre de Freud à Ferenczi en date du 6 décembre 1910, S. Freud et S. Ferenczi, Correspondance, t. 1, 1908-1914, Calmann-Lévy, 1992, groupe de traduction du Coq-Héron, p. 251. 